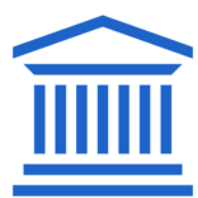 \\ GHENT \\ UNIVERSITY
}

\author{
biblio.ugent.be
}

The UGent Institutional Repository is the electronic archiving and dissemination platform for all UGent research publications. Ghent University has implemented a mandate stipulating that all academic publications of UGent researchers should be deposited and archived in this repository. Except for items where current copyright restrictions apply, these papers are available in Open Access.

This item is the archived peer-reviewed author-version of:

Fabrication and replication of high efficiency blazed gratings with grayscale electron beam lithography and UV nanoimprint lithography

M.-A. Mattelin, A. Radosavljevic, J. Missinne, D. Cuypers, S. Kommeren, J. Vandael, J. M. ter Meulen, L. Verduyckt, and G. Van Steenberge

In: Proceedings of SPIE, 11292, page 1129204 (11 pages), 2020.

https://doi.org/10.1117/12.2542171

To refer to or to cite this work, please use the citation to the published version:

M.-A. Mattelin, A. Radosavljevic, J. Missinne, D. Cuypers, S. Kommeren, J. Vandael, J. M. ter Meulen, L. Verduyckt, and G. Van Steenberge (2020). "Fabrication and replication of high efficiency blazed gratings with grayscale electron beam lithography and UV nanoimprint lithography." Proc. SPIE. 11292. 10.1117/12.2542171. 


\title{
Fabrication and replication of high efficiency blazed gratings with grayscale electron beam lithography and UV nanoimprint lithography
}

\author{
Marie-Aline Mattelin ${ }^{\mathrm{a}}$, Ana Radosavljevic ${ }^{\mathrm{a}}$, Jeroen Missinne ${ }^{\mathrm{a}}$, Dieter Cuypers $^{\mathrm{a}}$, Sander \\ Kommeren $^{\mathrm{b}}$, Jos Vandael ${ }^{\mathrm{c}}$, Jan Matthijs ter Meulen ${ }^{\mathrm{b}}$, Luc Verduyckt ${ }^{\mathrm{c}}$, and Geert Van \\ Steenberge $^{\mathrm{a}}$ \\ ${ }^{a}$ Center for Microsystems Technology (CMST), Ghent University - imec, 9052 Ghent, Belgium \\ b Morphotonics B.V., 5503LM Veldhoven, The Netherlands \\ ${ }^{\mathrm{c}}$ Zweko Optics BVBA, 3950 Kaulille, Belgium
}

\begin{abstract}
In a waveguide-type display for augmented reality, the image is injected in the waveguide and extracted in front of the eye appearing superimposed on the real world scene. An elegant and compact way of coupling these images in and out is by using blazed gratings, which can achieve high diffraction efficiencies, thereby reducing stray light and decreasing the required power levels. This study investigates the fabrication of blazed gratings with grayscale electron beam lithography and the subsequent replication of the realized 3D grating structures in a polymer material with ultraviolet nanoimprint lithography. As such, diffractive elements are realized on a waveguide sheet, with very good control over the dimensions and the profile of the printed features. Blazed gratings are designed for green light $(\lambda=543 \mathrm{~nm})$ and a diffraction angle of $43^{\circ}$. Making use of a PMMA resist and by carefully optimizing the electron-beam parameters, electron dose distributions and development step, blazed gratings with a pitch of $508 \mathrm{~nm}$ and a fill factor of 0.66 are achieved. Finally, a master is realized with two blazed gratings, $3 \mathrm{~cm}$ apart, which are replicated using ultraviolet nanoimprint lithography onto a waveguide sheet. The in- and outcoupling of an image through these two blazed gratings is shown, appearing sharp and non-distorted in the environment, and a throughput efficiency of $17.4 \%$ is confirmed.
\end{abstract}

Keywords: Waveguide-type head mounted display, blazed gratings, grayscale electron-beam lithography, ultraviolet nanoimprint lithography

\section{INTRODUCTION}

With the virtual reality (VR) and augmented reality (AR) application's boom, head-mounted displays (HMDs) are gaining a lot of attention. See-through HMDs have the capability of superimposing a virtual image on the real world scene without impairing the view of the user. The waveguide approach is of particular interest because the HMD can be made small and light-weighted. ${ }^{1-4}$ The general principle of a waveguide-type HMD is schematically displayed in figure 1. Light is coupled in a waveguide and coupled out in front of the eye after propagating in the waveguide by total internal reflection (TIR). As such, a virtual image or information is displayed in the field of view of the user. Some first commercial product appeared in the past few years, such as Google Glass, ${ }^{5}$ Epson BT- $350^{6}$ and Microsoft Hololens. ${ }^{7}$

Here, the main targeted application is the integration of a see-through HMD in a motorcycle helmet. This will enhance the motorcyclists road safety and experience. A display integrated in the helmet is safer and more ergonomic than a traditional screen on the dashboard where the motorcyclist has to look down. Furthermore, for safety reasons, the amount of (dangerous) components mounted inside the helmet or especially in front of the eye should be limited. This waveguide based HMD solution allows to project virtual information in the field of

Further author information: (Send correspondence to Marie-Aline Mattelin)

E-mail: mariealine.mattelin@ugent.be, Telephone: 003292645370 


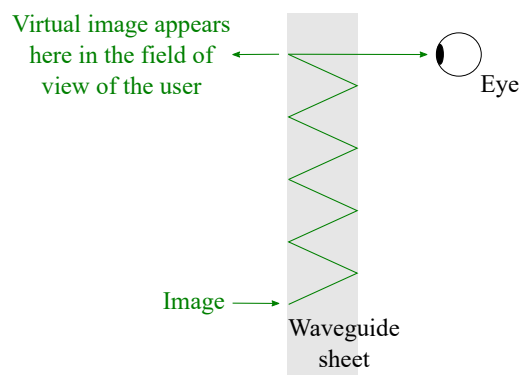

Figure 1: Schematic representation of a waveguide-type HMD.

view of the user, such as safety, performance and mobility data, while the optical engine, generating the image information, can be mounted further away, for example at the outside of the helmet. The only component in front of the eye is the transparent waveguide sheet. Using blazed gratings as diffractive elements, a high optical efficiency can be achieved and the power consumption can be reduced.

In this paper, we investigate the possibilities of fabricating and replicating high efficient blazed gratings. Using grayscale electron-beam lithography (EBL) multilevel structures can be fabricated which can be reflowed into continuous slopes by thermally activated selective topography equilibration (TASTE) ${ }^{8-10}$ to achieve rounded and blazed structures. We examine the feasibility of a fabrication process without thermal reflow ${ }^{11}$ as we want to limit the process steps to achieve a well-controlled fabrication flow. To integrate the blazed gratings in a waveguide-type HMD, a suitable replication process is needed to copy the grating structures from a master mold to a waveguide sheet. Polymers offer the possibility of replicating gratings by imprinting with a potentially high cost efficiency. A stamp-based imprinting process allows the definition of nanostructures with very good control over the dimensions and the profile of the printed features. ${ }^{12,13}$ The use of ultraviolet nanoimprint lithography (UV-NIL) is already reported for replicating a 3D pyramid-shaped array. ${ }^{14}$ This process is also chosen here because it can be scaled to roll-to-roll or roll-to-plate high-throughput manufacturing. ${ }^{15,16}$

We design blazed gratings for green light $(\lambda=543 \mathrm{~nm})$ and a diffraction angle of $43^{\circ}$. We completely describe the fabrication with grayscale electron-beam lithography and the subsequent replication in a polymer waveguide using ultraviolet nanoimprint lithography. We evaluate the achieved structures by investigating the cross sections with FIB SEM and by measuring the diffraction efficiencies to optimize the process flow. The final gratings can be implemented directly in the intended application. We show that an image injected in the waveguide and extracted in front of the eye appears sharp and non-distorted superimposed on the real world scene and confirm a throughput efficiency of $17.4 \%$.

\section{BLAZED GRATING DESIGN}

Figure 2 shows the optical design layout of an HMD consisting of a waveguide for image transmission, surface relief gratings for in- and outcoupling of an image to the waveguide and an optical engine used to generate the image which is transmitted through the waveguide. A lens is added to collimate the image before incoupling. The waveguide material is PMMA (poly methyl methylacrelate) with a $30 \mu \mathrm{m}$-thick OrmoCore layer (Microresist Technology, Berlin, Germany) on top of which the gratings are imprinted.

Both gratings are imprinted on the same side of the waveguide sheet for easier fabrication. For practical reasons, the light engine is also on this side. Hence, the incoupling grating should work as a transmission grating, while the outcoupling grating should be a reflection grating. Light propagates between the in- and outcoupling gratings in the waveguide due to TIR at the polymer/air interfaces. The transmission incoupling grating should diffract most of the energy of the normally incident light from the light source to the first diffraction order $m=-1$. This first diffraction order must be larger than the critical angle for TIR, as is shown in figure 3(a). At the 


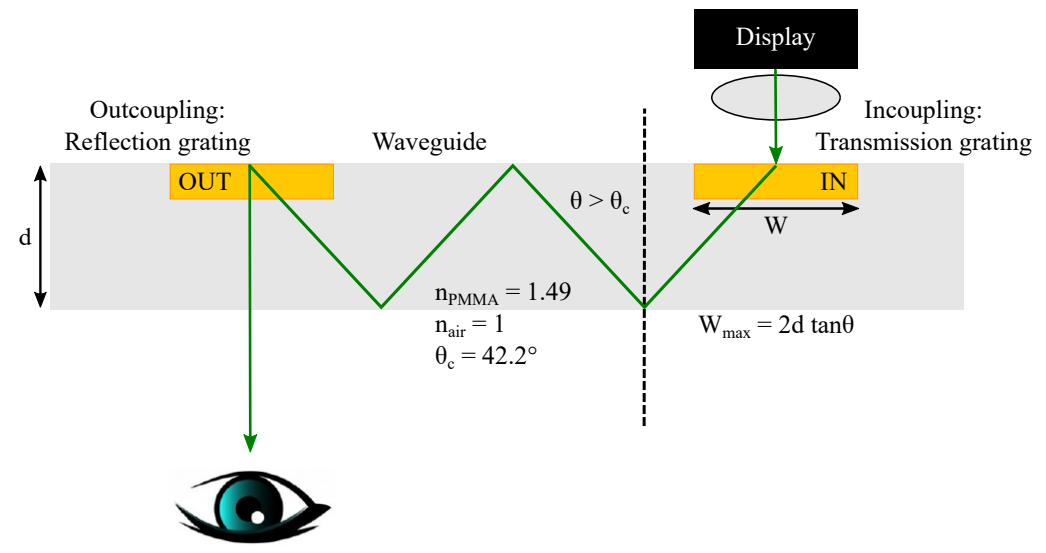

Figure 2: Optical design layout of a waveguide-type HMD with gratings.

outcoupling grating, most of the energy of the light coming under an angle should be reflected towards the eye to the zeroth order, this is illustrated in figure 3(b).
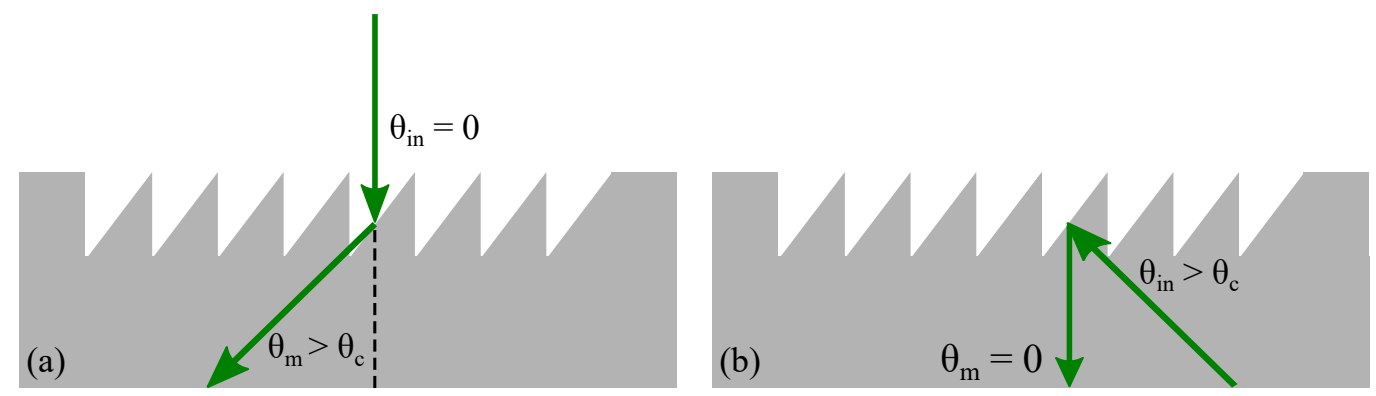

Figure 3: Schematic representation of the (a) input transmission and (b) output reflection blazed gratings. The green arrows show the light path.

Under normal light conditions, human eyes are most sensitive to a yellowish-green color. ${ }^{17}$ Therefore, green light $(\lambda=543 \mathrm{~nm})$ is used for projecting the images. At this wavelength, the refractive indices of PMMA and OrmoCore are, respectively, 1.49 and 1.565 and the minimal angle for TIR at the PMMA/air interface is $42.2^{\circ}$. To avoid backcoupling of incoupled light, the maximal length of the incoupling grating is $W_{\max }=2 d \tan \theta$, where $d$ is the waveguide thickness and $\theta$ is the diffraction angle. For a diffraction angle of $43^{\circ}$ and a waveguide thickness of $5 \mathrm{~mm}$, images of maximum $9.3 \mathrm{~mm}$ broad can be launched $\left(W_{\max }=9.3 \mathrm{~mm}\right)$.

The diffraction angle depends both on the wavelength of the incoupled light and on the grating period and is defined by the grating equation. The efficiency of the gratings with a fixed period depends on the profile of the grating and can be calculated numerically. Therefor we use the finite difference time domain (FDTD) method, implemented in the commercially available FDTD software (Lumerical Inc., Vancouver, BC, Canada). The grating length of $5 \mathrm{~mm}$ is impractical to be directly applied in simulation. Hence, only one grating period is simulated and periodic boundary conditions are applied. These boundary conditions imply that the grating length is infinite. We can state that this is a valid assumption in our case as the number of periods in a $5 \mathrm{~mm}$ long grating is very large. In the vertical direction the simulation window is $4 \mu \mathrm{m}$ high, so the simulation only runs in the $30 \mu \mathrm{m}$-thick OrmoCore layer and does not take the substrate into account. In figure 4 the grating period and the number of periods in a $5 \mathrm{~mm}$ long first order diffraction grating in OrmoCore are shown as a function of the diffraction angle for a wavelength of $543 \mathrm{~nm}$. For a diffraction angle of $43^{\circ}$ the grating period is $508 \mathrm{~nm}$.

Since we do not reflow the e-beam structures, the grating profile can deviate from a smooth triangular shape 

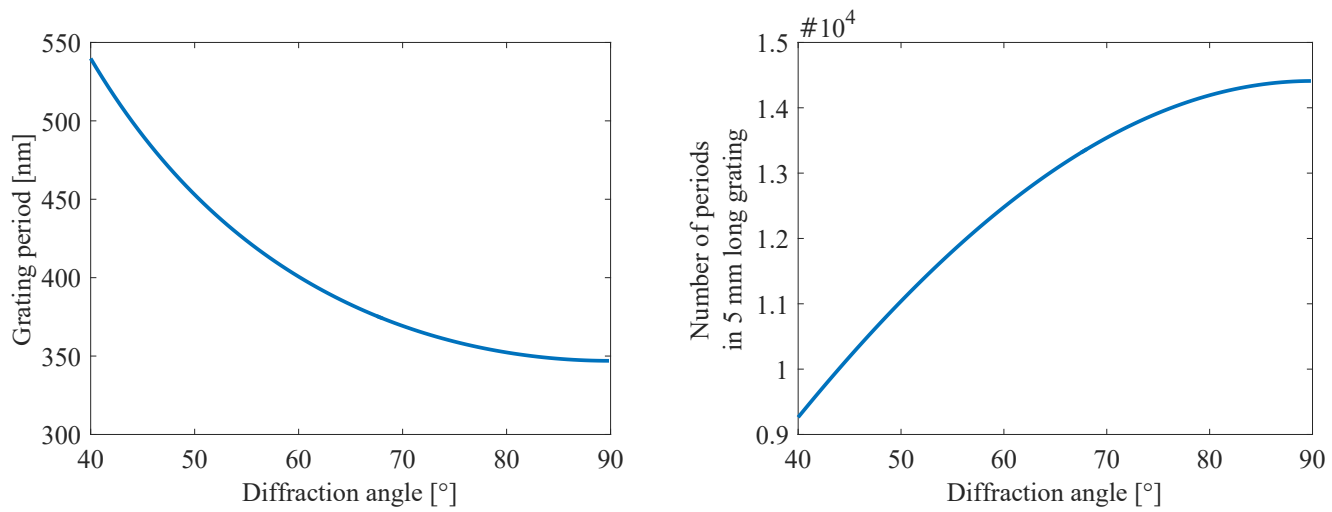

Figure 4: The grating period and the number of periods in a $5 \mathrm{~mm}$ long first order diffraction grating in OrmoCore as a function of the diffraction angle for a wavelength of $543 \mathrm{~nm}$.

although the EBL parameters are carefully optimized to suppress deviations as much as possible. During the fabrication it was noticed that the gratings tend to have a triangular shape with an extra top. This shape is shown in figure 5. $\Delta$ is the pitch, $H$ is the total height and $D$ is the total width, defined as $D=F F * \Delta$. The top is characterized by $H_{1}$ : the height of the grating without the top, and $D_{\text {top }}$ : the width of the grating top which is defined in $\psi=\frac{D-D_{t o p}}{D}$.
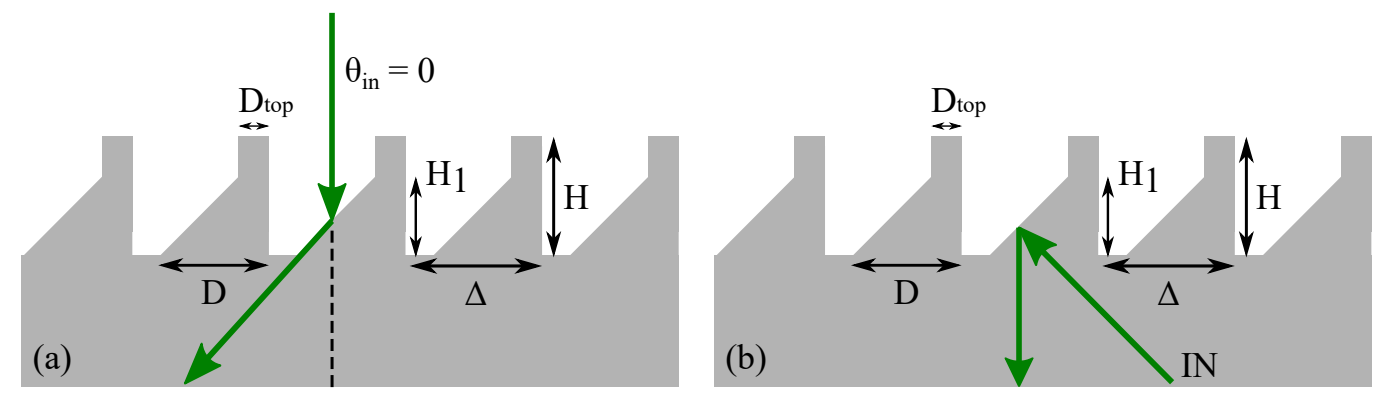

Figure 5: Schematic representation of the (a) input transmission and (b) output reflection blazed gratings with top.

The efficiencies of such in- and outcoupling gratings in OrmoCore as a function of the two parameters $H_{1}$ and $\psi$ are simulated for a grating pitch $\Delta$, grating height $H$ and width $D$ of $508 \mathrm{~nm}, 480 \mathrm{~nm}$ and $300 \mathrm{~nm}$, respectively, and are shown in figure 6 . For the incoupling grating there is a broad range of parameters leading to a grating with an efficiency of more than $55 \%$. The parameters corresponding to the maximal achievable efficiency are $H_{1}=300 \mathrm{~nm}$ and $\psi=0.7$. For the outcoupling grating the parameters for the maximal achievable efficiency are $H_{1}=380 \mathrm{~nm}$ and $\psi=1$.

\section{FABRICATION METHODS}

To realize a waveguide-type HMD with blazed gratings, first a master mold with two blazed gratings is realized using grayscale EBL which is then replicated onto a waveguide sheet using UV-NIL. EBL allows 3D patterning by using dose-modulated exposure and wet development. To realize the triangular grating profile different grating lines are exposed with a different dose in a sensitive and high-contrast EBL resist. For the replication of the master mold two replication steps are involved, as is shown in figure 8. In the first imprint step the inverse shape of the master mold is replicated into a soft mold. In the second step the soft mold is rolled over the polymer material to realize the grating structures on the waveguide substrate. 
(a)

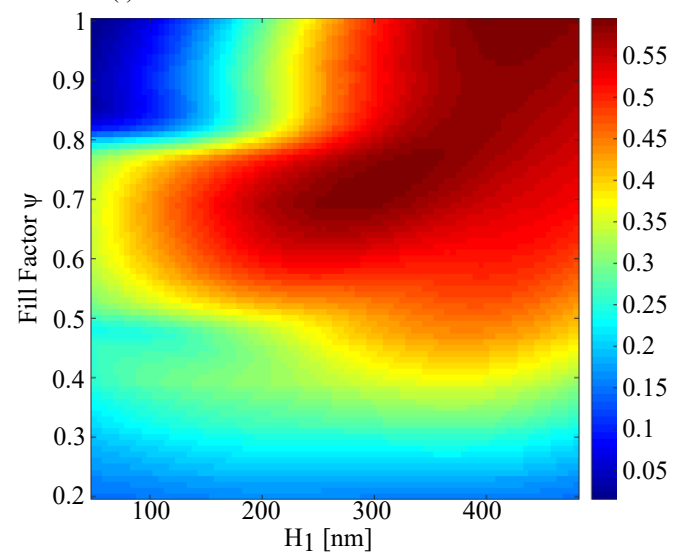

(b)

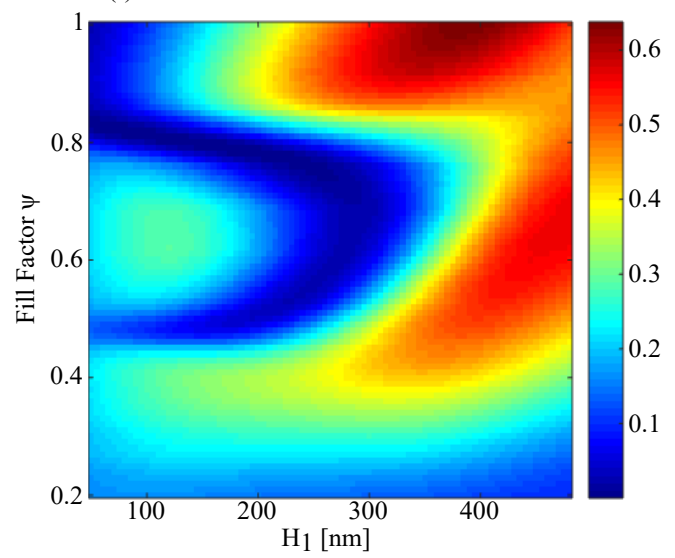

Figure 6: Efficiency of blazed gratings with top: (a) Input transmission grating: efficiency of power transmission to the first diffraction order. (b) Output reflection grating: efficiency of power reflection to the zeroth diffraction order.

\subsection{Master fabrication using grayscale electron-beam lithography}

950 PMMA A7 (MicroChem, Westborough, MA, USA) is used as EBL resist. A 4" Si wafer is treated with plasma (Diener Pico, $190 \mathrm{~W} 40 \mathrm{kHz}$ generator, $24 \mathrm{~s}, 0.8$ mbar, gas used: air) and spin coated with 950 PMMA A7 $(1500 \mathrm{rpm}, 45 \mathrm{~s}$, coating thickness $=850 \mathrm{~nm})$. A soft baking step is applied $\left(150^{\circ} \mathrm{C}, 90 \mathrm{~s}\right)$ before AR-PC 5090 (Allresist, Strausberg, Germany), a conductive protective coating, is spin coated (2000 rpm, $60 \mathrm{~s}$, coating thickness $=60 \mathrm{~nm})$ and soft baked $\left(90^{\circ} \mathrm{C}, 120 \mathrm{~s}\right)$. The resist is exposed in a Raith Voyager EBL system with a voltage acceleration of $50 \mathrm{keV}$. A small beam current of $0.41-0.5 \mathrm{nA}$ is selected to improve the resolution. A grating of several $\mathrm{mm}^{2}$ is exposed by stitching multiple writefields of $200 \mathrm{x} 500 \mu \mathrm{m}^{2}$. Within one writefield it is the electron-beam which scans the area to be exposed, ensuring a very good control over the patterning. Between different writefields it is the stage which moves, eventually leading to stitching errors. Several accurate alignment procedures before the exposure can minimize these errors. One writefield is scanned in multiple passes; in each pass a grating is exposed with the same pitch but with a different fill factor and dose. This is schematically shown in figure 7. A different dose results in a different resist thickness after development. After the exposure, the wafer is rinsed with DI water to remove the conductive coating, developed in MIBK:IPA 1:3 for 20-40 s, rinsed in IPA and blown dry with a nitrogen gun. Cross section inspection of the grating profile is done with a focused ion beam (FIB) scanning electron microscope (SEM) (Nova 600 NanoLab, FEI Company, Hillsboro, OR, USA) and reveals the desired relief structure. Multiple cycles of EBL and cross section inspection were needed to optimize the e-beam writing parameters, electron-dose distributions and fill factors of the different grating layers.

\subsection{Replication of the gratings on waveguides using UV-NIL}

The transfer of the blazed gratings on the master mold to the final waveguide sheet is done using two UV-NIL steps and is schematically shown in figure 8. First, a soft stamp is fabricated by replicating the master mold into a UV-curable transparent perfluoropolyether (PFPE) polymer. This polymer is prepared by adding $3 \%$ photoinitiator to working stamp material EVGNIL UV/AF1 (EV Group, St. Florian am Inn, Austria) by weight. After manually mixing thoroughly, the viscous mixture is let to rest for $60 \mathrm{~min}$ for degassing. Subsequently, the mixture is spin coated at a slow speed $(500 \mathrm{rpm}, 60 \mathrm{~s})$ to achieve a relatively thick but homogeneous layer on the master mold. The material is covered with a PET foil and the stack is UV exposed (30 $\left.\frac{\mathrm{mW}}{\mathrm{cm}^{2}}, 60 \mathrm{~s}\right)$. Afterwards, the stack is peeled off and the structures are reverse copied in the PFPE material. This soft stamp is now used for imprinting the structures in the final polymer material on the waveguide substrate. OrmoCore (Microresist Technology, Berlin, Germany) is spin coated on a clean and plasma treated (Diener Pico, $190 \mathrm{~W} 40$ $\mathrm{kHz}$ generator, $24 \mathrm{~s}, 0.8$ mbar, gas used: air) PMMA substrate (3000 rpm, $30 \mathrm{~s}$, coating thickness $=30 \mu \mathrm{m})$. The soft stamp is brought in contact with the OrmoCore coating in a rolling motion to avoid air being trapped. 
(a)

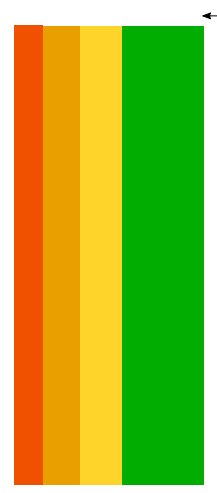

$\operatorname{Pitch} \Lambda$

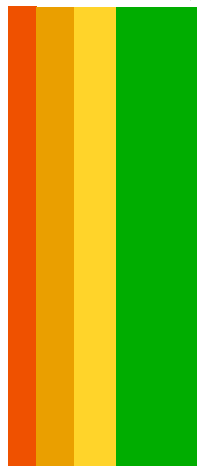

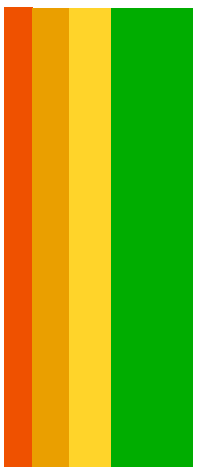

(b)

1)

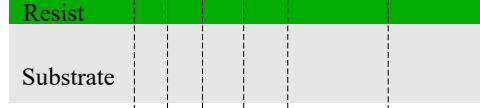

2)

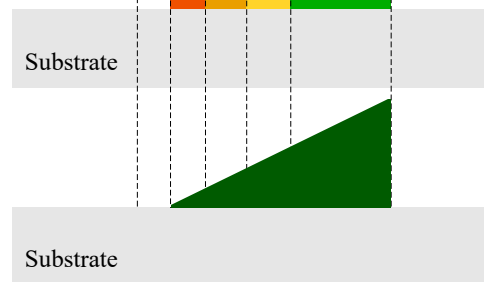

Figure 7: Schematic overview of the e-beam exposure strategy with multiple grating layers. Each grating layer is exposed with a different dose: $D_{1}$ being the highest and $D_{4}$ being the lowest, between $D_{4}$ from one grating period and $D_{1}$ from the next grating period there is an unexposed region. (a) Shows the top view of a grating with different layers, (b) shows the side view: 1) dose-modulated exposure 2) estimation of the remaining resist thickness according to the contrast curve 3 ) actual grating profile.

This stack is UV exposed (30 $\left.\frac{m W}{c m^{2}}, 60 \mathrm{~s}\right)$ after which the soft stamp is manually peeled off.
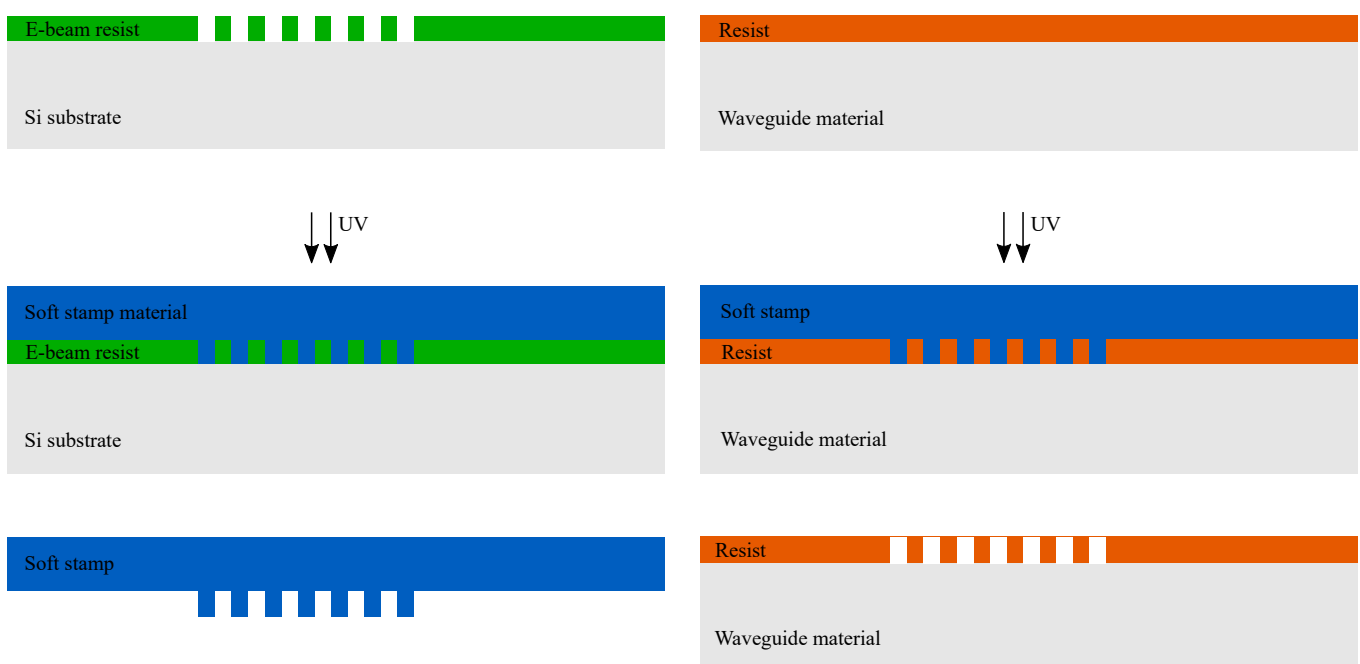

Figure 8: Schematic overview of the two-step imprinting process.

Using the Roll-to-Plate imprint technology developed by Morphotonics, micro- and nanostructures can be applied onto discrete substrates on a large scale. Also in this process the required optical structures are replicated by the use of a flexible stamp. This flexible stamp has the inverse shape of the desired structures on its surface. The imprint process is depicted in figure 9 .

The actual imprint process starts by dispensing a liquid UV-curable lacquer on the substrate. Next, the flexible stamp is laminated on the lacquer-coated substrate. By using a specific roller and pressure control, air inclusions are prevented and a uniform layer is created. After lamination, the lacquer is cured with UV light applied 
through the flexible stamp. In this stage, the lacquer is formed into its final shape and the flexible stamp will be delaminated smoothly by again using a specific roller and pressure control. After delamination, the flexible stamp is indexed to the start position, after which the imprint process can start again. The Morphotonics technology can process a wide range of structure shapes and sizes and is ideally suited to imprint structures ranging from hundreds of micrometers down to 50 nanometer.

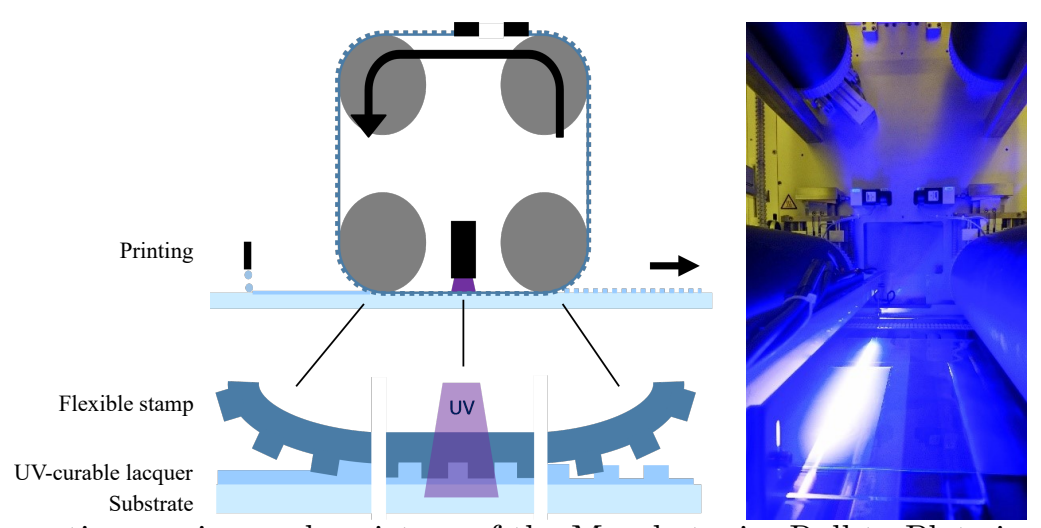

Figure 9: Schematic overview and a picture of the Morphotonics Roll-to-Plate imprint process.

The benefit of the Morphotonics Roll-to-Plate imprint technology is twofold. The imprint technology is costeffective because of the re-usable flexible stamp and the Roll-to-Plate imprint method is scalable, enabling the imprinting of extreme large areas of more than $0.5 \times 0.5 \mathrm{~m}^{2}$. The ability to imprint large areas gives the opportunity to add light management structures to large products (i.e. large displays or solar panels) or it can be used to increase the throughput: multiple small products (i.e. for helmets) can be imprinted in one replication cycle. Volumes of above 1M products per year can be achieved by this Roll-to-Plate imprint technology, making it an interesting mass production method.

\section{RESULTS AND DISCUSSION}

\subsection{The blazed grating profile}

The grayscale EBL and UV-NIL processes are optimized until a blazed grating profile is realized that could achieve a diffraction efficiency of $50 \%$, according to simulations. The cross section made with FIB SEM of this imprinted grating profile is shown in figure 10. For this grating it takes $7 \mathrm{~min}$ to write one writefield of 200x500 $\mu \mathrm{m}^{2}$. Hence, a grating area of $3 \times 2 \mathrm{~mm}^{2}$ is chosen to have an acceptable e-beam writing time for fabricating two blazed gratings, $3 \mathrm{~cm}$ apart, on one master.

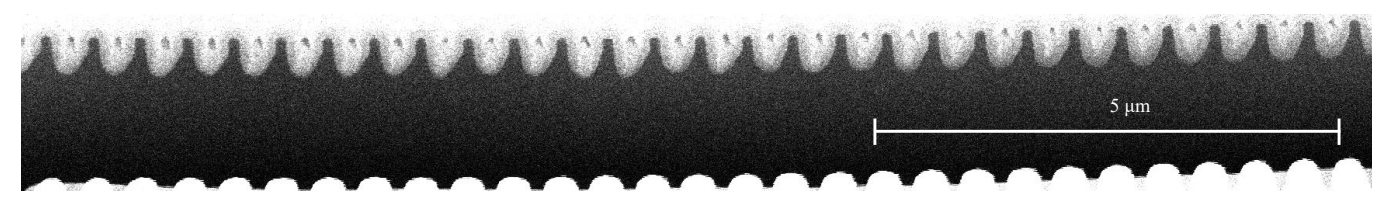

Figure 10: FIB SEM image of the imprinted blazed grating with a simulated incoupling efficiency of $50 \%$.

\subsection{Optical measurements and demonstration}

To quantify the diffraction efficiency, the master with two blazed gratings of $3 \times 2 \mathrm{~mm}^{2}$ is imprinted in OrmoCore on 5-mm-thick PMMA. A picture of the master and the imprinted grating can be found in figure 11. As a first experiment, the amount of light that is coupled in by one grating is measured. Therefor, two $45^{\circ}$ facets are polished at the PMMA waveguide sheet. Figure 12 shows the measurement setup: the grating is illuminated 
with green laser light (543.5 nm, Helium-Neon Laser 1652, JDS Uniphase, Milpitas, CA, USA), polarized parallel to the grating lines, and the power is measured with a detector (Newport Model 818-SL) connected to a power meter (Newport 1930C optical power meter, Newport Corporation, Irvine, CA, USA). The TIR is visible in the PMMA sample on the picture of the setup in figure 12.
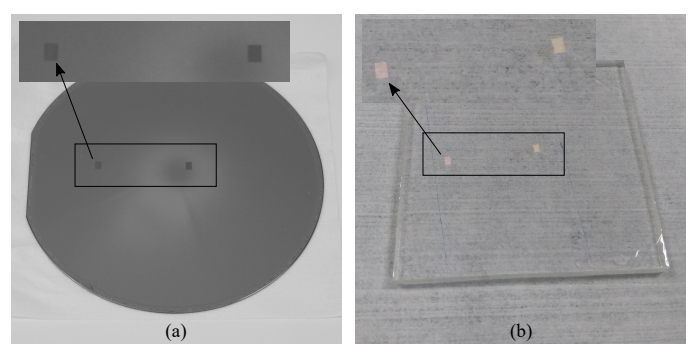

Figure 11: (a) The master with blazed gratings in PMMA on Si and (b) the imprint of the blazed gratings in OrmoCore on 5-mm-thick PMMA.
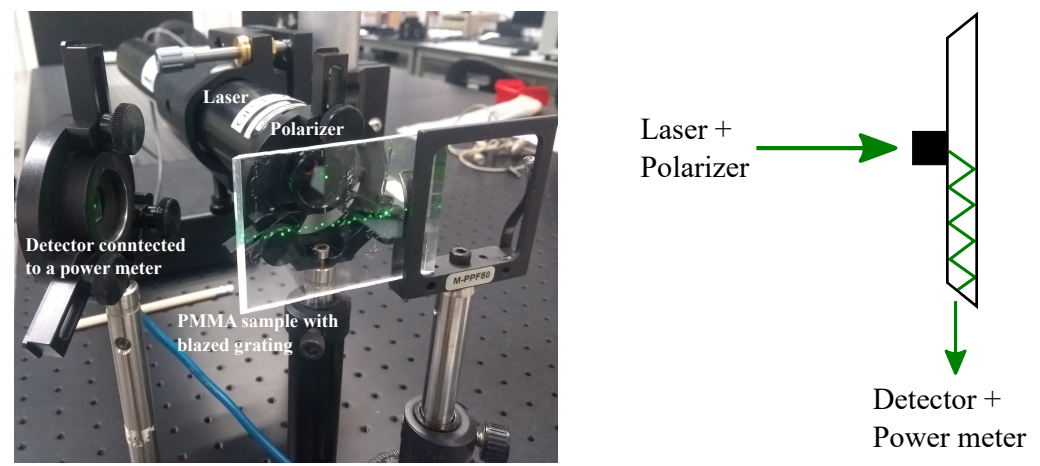

Figure 12: A picture and a schematic of the optical setup for diffraction efficiency measurements.

Parallel polarized green laser light illuminates the grating perpendicularly and the power is measured at both facets and in the zeroth diffraction order. Simulations showed that most of the light is diffracted in the direction where the slope is oriented to, which is $50 \%$ for this grating profile. This is confirmed with the measurements: $48.4 \%$ of the incident parallel polarized light is coupled out at the facet where the slope is oriented to, while only $16.1 \%$ is coupled out at the other facet and $26.8 \%$ is recorded at the zeroth diffraction order. As a comparison, the same measurement is done for a symmetric binary grating. In this case $28.1 \%$ and $26.4 \%$ are coupled to the facets while $45.2 \%$ is transmitted to the zeroth diffraction order. The diffraction efficiency to the first order is significantly lower for the binary grating while the amount of light coupled to the zeroth order is almost twice as high.

For the second experiment, green laser light illuminates the first grating perpendicularly and the light is coupled in the slab waveguide where it propagates due to TIR until it is coupled out by the second grating. The throughput is measured. This is the ratio between the intensity of the outcoupled light and the intensity of the light incident on the input grating. In figure 14 a throughput of $17.4 \%$ is measured for blazed gratings when parallel polarized light is following the green path in figure 14, while only $3.23 \%$ is measured when the light follows the red path. In both cases also the light coupled to the zeroth diffraction order is measured, the values for the green and red light path are $30.3 \%$ and $43.6 \%$, respectively. Again, a comparison with binary gratings is made. In one direction a throughput of $2.74 \%$ and a zeroth order diffraction efficiency of $50.0 \%$ is measured. In the other direction a throughput of $4.19 \%$ and a zeroth order diffraction efficiency of $45.8 \%$ is measured. Also this experiment proves that the blazed gratings are more efficient structures for perpendicular incident light. 


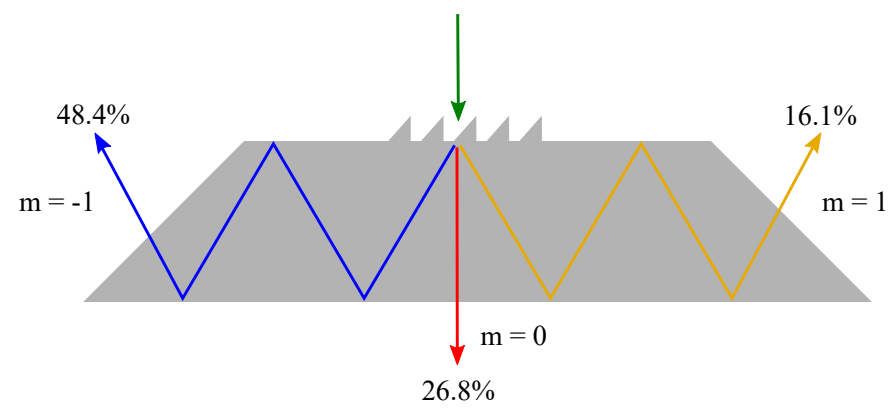

Figure 13: The measured power at both $45^{\circ}$ polished facets and in the zeroth order when the blazed grating is illuminated by parallel polarized green laser light.

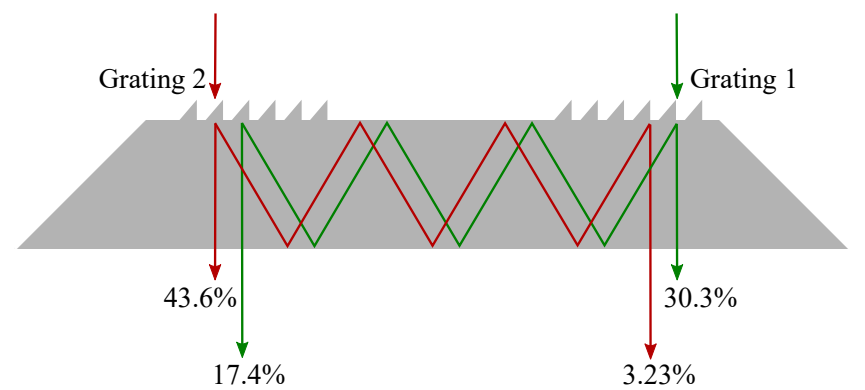

Figure 14: The measured throughput and diffraction efficiencies to the zeroth diffraction order for two blazed gratings for parallel polarized green laser light.

A modified picoprojector (UO smart beam laser portable mini projector, SK Telecom, Seoul, South Korea) was used to demonstrate the HMD principle. For this demo, the lens in the picoprojector was replaced by a combination of 2 other lenses, i.e. a plano concave lens with a focal length of $-50 \mathrm{~mm}$ and a plano convex lens with a focal length of $40 \mathrm{~mm}$, to enhance the collimation of the light beam. The power coming out of the picoprojector was attenuated 1000 times. A 5x6 chess pattern of green and black squares was projected on the PMMA sample and perceived as a virtual image behind the waveguide. The perceived AR image, coupled in- and out by blazed gratings in figure 15, appeared clear and sharp on the environment and the stitching errors were not visible in the pattern. The image appeared in focus at $4 \mathrm{~m}$ in front of the waveguide. This distance can be tuned by varying the distance between the two lenses in front of the picoprojector. The fact that the image is quite small and not sharp at the edges is a result of the limited grating area and can be improved by writing larger gratings.

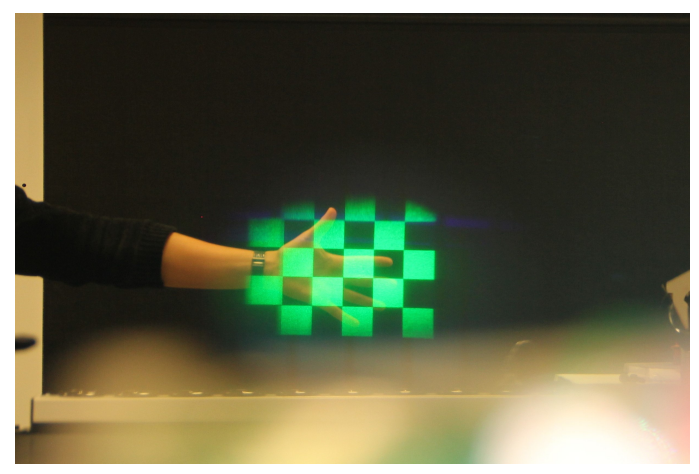

Figure 15: The chess pattern projected by blazed gratings on the environment. 


\section{CONCLUSION}

In this paper we demonstrated a waveguide-type HMD with blazed gratings, which are 4 times more efficient than binary gratings. First, a blazed grating profile was designed to achieve an in- and outcoupling efficiency of $55 \%$. EBL was used to fabricate these structures by modulating the electron-dose over the grating pitch. After several optimization cycles, a grating profile was achieved that could achieve an incoupling efficiency of $50 \%$, according to simulations. Two of such gratings were fabricated, $3 \mathrm{~cm}$ apart, on one master with EBL and afterwards imprinted with UV-NIL on a waveguide sheet. These blazed gratings proved to be very efficient: an incoupling efficiency of $48.4 \%$ and a throughput efficiency of $17.4 \%$ were recorded. Finally, it could be shown that an image injected in the waveguide and extracted in front of the eye appeared sharp and non-distorted superimposed on the real world scene. By using these efficient blazed grating structures the required power levels can be limited when the whole system is integrated in a helmet.

\section{Acknowledgments}

This research was funded within the imec.icon project smartGLAZ, bringing academia and industry together (Flanders Innovation Entrepreneurship project nr. HBC.2017.0634). We want to thank Regan Watts and Jochen Vleugels from the University of Antwerp for providing us with the picoprojector. We also want to thank Lothar Mader from IMEC for polishing the PMMA samples.

\section{Disclosures}

The authors declare no conflicts of interest.

\section{References}

[1] V. Brac de la Perrière, "Understanding waveguide-based architecture and ways to robust monolithic optical combiner for smart glasses," Proc. SPIE 10676, 106761D (2018).

[2] C. Pan, Z. Liu, Y. Pang, X. Zheng, H. Cai, Y. Zhang and Z. Huang, "Design of a high-performance incoupling grating using differential evolution algorithm for waveguide display," Opt. Express 26(20), 26646$26662(2018)$.

[3] M.-L. Piao, S.-K. Gil and N. Kim, "Design of wide angle holographic waveguide monocular head-mounted display using photopolymer," Proc. SPIE 9386, 93860K (2015).

[4] J. Guo, Y. Tu, L. Yang, L. Wang and B. Wang, "Holographic waveguide display with a combined-grating in-coupler," Applied Optics 55(32), 9293-9298 (2016).

[5] Google, https://www.google.com/glass/start/

[6] Epson, https://www.epson.be/products/see-through-mobile-viewer/moverio-bt-350\#specifications

[7] Microsoft, https://www.microsoft.com/en-us/hololens

[8] A. Schleunitz, V.A. Guzenko, M. Messerschmidt, H. Atasoy, R. Kirchner and H. Schift, "Novel 3D micro- and nanofabrication method using thermally activated selective topography equilibration (TASTE) of polymers," Nano Convergence 1, 7 (2014).

[9] A. Schleunitz and H. Schift, "Fabrication of 3D nanoimprint stamps with continuous reliefs using dosemodulated electron beam lithography and thermal reflow," J. of Micromechanics and Mircoengineering 20(9), $095002(2010)$.

[10] R. Kirchner, V.A. Guzenko, I. Vartiainen, N. Chidambaram and H. Schift, "ZEP520A - a resist for electronbeam grayscale lithograpy and thermal reflow," Microelectron. Eng. 153, 71-76 (2016). 
[11] T. Shiono, T. Hamamoto and K. Takahara, "High-efficiency blazed diffractive optical elements for the violet wavelength fabricated by electron-beam lithography," Appl. Opt. 41(13), 2390-2393 (2002).

[12] J. Missinne, N. Teigell Benéitez, M.-A. Mattelin, A. Lamberti, G. Luyckx, W. Van Paepegem and G. Van Steenberge, "Bragg-grating-based photonic strain and temperature sensor foils realized using imprinting and operating at very near infrared wavelengths," Sensors 18(8), 2717 (2018).

[13] J. Missinne, N. Teigell Benéitez, A. Lamberti, G. Chiesura, G. Luyckx, M.-A. Mattelin, W. Van Paepegem and G. Van Steenberge, "Thin and flexible polymer photonic sensor foils for monitoring composite structures," Adv. Eng. Mater. 20(2), 1701127 (2018).

[14] K. Mohamed, M.M. Alkaisi and R.J. Blaikie, "A three-dimensional ultraviolet curable nanoimprint lithography (3D UV-NIL)," AIP Conf. Proc. 1151, 114 (2009).

[15] N. Kooy, K. Mohamed, L.T. Pin and O.S. Guan, "A review of roll-to-roll nanoimprint lithography," Nanoscale Res. Lett. 9, 320 (2014).

[16] S.H. Ahn and L.J. Guo, "Large-area roll-to-roll and roll-to-plate nanoimprint lithography: a step toward high-throughput application of continuous nanoimprinting," ACS Nano 3(8), 2304-2310 (2009).

[17] NDT Resource Center, "The human's eye response to light," https://www.ndeed.org/EducationResources/CommunityCollege/PenetrantTest/Introduction/lightresponse.htm 